The Astrophysical Journal, 651: L29-L32, 2006 November 1

(C) 2006. The American Astronomical Society. All rights reserved. Printed in U.S.A.

\title{
SUBSTRUCTURE IN TIDAL STREAMS: TRIBUTARIES IN THE ANTICENTER STREAM

\author{
C. J. Grillmair
} \\ Spitzer Science Center, 1200 East California Boulevard, Pasadena, CA 91125; carl@ipac.caltech.edu Received 2006 August 18; accepted 2006 September 14; published 2006 October 10
}

\begin{abstract}
We report on the detection in Sloan Digital Sky Survey data of at least three, roughly parallel components in a $65^{\circ}$-long, stellar stream complex previously identified with the Monoceros Ring. The three-stream complex varies in width from $4^{\circ}$ to $6^{\circ}$ along its length and appears to be made up of two or more narrow substreams as well as a broader, diffuse component. The width and complexity of the stream indicate that the progenitor was likely a dwarf galaxy of significant size and mass. The stream is $8.9 \mathrm{kpc}$ distant and is oriented almost perpendicularly to our line of sight. The visible portion of the stream does not pass near any known dwarf galaxies, and a preliminary orbit does not point to any viable progenitor candidates. Orbits for the narrower substreams can be modeled with velocity offsets from the broad component of $\approx 8 \mathrm{~km} \mathrm{~s}^{-1}$. We suggest that the broad component is likely to be the remains of a dwarf galaxy, while the narrower streams constitute the remnants of dynamically distinct components that may have included a native population of globular clusters. While the color of the main-sequence turnoff is not unlike that for the Monoceros Ring, neither the visible stream nor any reasonable projection of its orbit passes through Monoceros or Canis Major, and we conclude that this stream is probably unrelated to the overdensities found in these regions.
\end{abstract}

Subject headings: Galaxy: halo — Galaxy: structure

\section{INTRODUCTION}

The value of large-scale digital sky surveys to studies of Galactic structure and the Local Group has become abundantly clear in recent years, and particularly in the last few months. In addition to the large-scale features attributed to past galaxy accretion events (Yanny et al. 2003; Majewski et al. 2003; Rocha-Pinto et al. 2004), Sloan Digital Sky Survey (SDSS) data were used to detect the remarkably strong tidal tails of Palomar 5 (Odenkirchen et al. 2001, 2003; Rockosi et al. 2002; Grillmair \& Dionatos 2006a), NGC 5466 (Belokurov et al. 2006a; Grillmair \& Johnson 2006), and streams due to extant or extinct globular clusters (Grillmair \& Dionatos 2006b) and dwarf galaxies (Belokurov et al. 2006b; Grillmair 2006). Willman et al. (2005), Zucker et al. (2006), and Belokurov et al. (2006c) recently used SDSS data to discover several new dwarf satellites of the Milky Way.

In this Letter we continue our analysis of the SDSS database to search for extended structures in the Galactic halo. We briefly describe our analysis in $\S 2$. We discuss the structure of a stream complex, attributed to the Monoceros Ring by Belokurov et al. (2006b) in $\S 3$, estimate distances in $\S 3.1$, and put constraints on the orbit in $\S 3.2$.

\section{DATA ANALYSIS}

Data comprising ugriz photometry for stars in the region $108^{\circ}<\alpha<162^{\circ}$ and $-4^{\circ}<\delta<68^{\circ}$ were extracted from Data Release 5 of the SDSS using the SDSS CasJobs query system. The data were analyzed using the matched filter technique employed by Grillmair \& Johnson (2006), Grillmair \& Dionatos (2006a, 2006b), Grillmair (2006), and described in detail by Rockosi et al. (2002). This technique is made necessary by the fact that, over the magnitude range and over the region of sky we are considering, the foreground disk stars outnumber the more distant stars in the Galactic halo by some 3 orders of magnitude. Applied in the color-magnitude domain, the matched filter is a means by which we can optimally differentiate between two populations.
We used the SDSS photometry to create a color-magnitude density or Hess diagram for both stars of interest and for the foreground population. Dividing the former by the latter, we generated an array of relative weights which we used as an optimal color-magnitude filter. We generated the search filter using the color-magnitude distribution of stars in M13. Owing to M13's proximity, we have a better measure of its luminosity function and the effects of SDSS completeness than we do for other objects in the Data Release 5 (DR5) field. We used all stars with $15<g<22.5$. We dereddended the SDSS photometry as a function of position on the sky using the DIRBE/ IRAS dust maps of Schlegel et al. (1998). A single Hess diagram for field stars was generated using $1.2 \times 10^{7}$ stars spread over $\sim 2200 \mathrm{deg}^{2}$ of DR5. We applied the M13 filter to the entire survey area, and the resulting weighted star counts were summed by location on the sky to produce a two-dimensional probability map.

In Figure 1 we show the final filtered star count distribution, using a filter matched to the $g, g-i$ color magnitude distribution of stars in M13 but shifted faintward by 0.3 mag. An exponential surface function has been subtracted from the image to remove the rapid rise in the number of disk stars at low Galactic latitudes, and a fourth-order polynomial surface has been used to remove remaining large-scale gradients. The final image has been binned to a pixel size of 0.08 and smoothed using a Gaussian kernel with $\sigma=0.2$.

\section{DISCUSSION}

Apparent in Figure 1 is a broad and complex stream running from north to south across the field. Portions of this stream are visible in Newberg et al. (2002) and Belokurov et al. (2006b). The narrow, curved stream running toward the northeast is part of the $63^{\circ}$-long globular cluster stream found by Grillmair \& Dionatos (2006a), hereafter referred to as GD-1. The broad, east-west stream just above the main gap in the data is the Sagittarius stream discussed by Belokurov et al. (2006b), although somewhat muted by our filtering due to its greater distance. The stream complex of interest extends from (R.A., decl.) $=\left(126^{\circ} 4,-0^{\circ} .7\right)$ to 


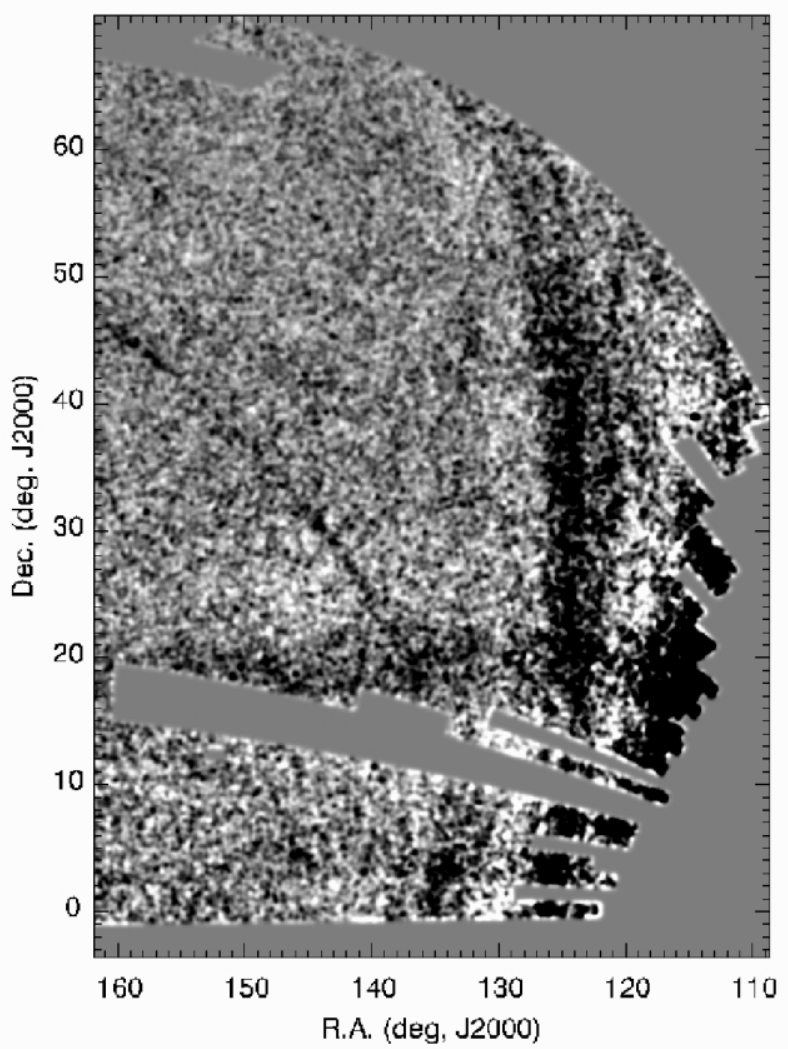

FIG. 1.-Smoothed, summed weight image of the SDSS field after subtraction of both an exponential and a fourth-order polynomial surface fit. Darker areas indicate higher surface densities. The weight image has been smoothed with a Gaussian kernel with $\sigma=0.2$. The solid gray areas are either data missing from DR5, or clusters or bright stars which have been masked out prior to analysis. The stream complex runs from (R.A., decl.) $=\left(126^{\circ} 4,-0^{\circ} 7\right)$ to approximately (R.A., decl. $)=\left(133^{\circ} 9,64^{\circ} .2\right)$. The feature at (R.A., decl. $)=\left(143^{\circ}, 30^{\circ}\right)$ is a portion of the cold stellar stream discovered by Grillmair \& Dionatos (2006a).

(R.A., decl. $)=\left(133^{\circ} .9,64^{\circ} .2\right)$ and runs in a $65^{\circ}$, nearly great circle path from Ursa Major in the north to Hydra in the south. The stream is truncated at both the southern and northern ends by the limits of the available data. We note that there is also an apparent concentration of stars at (R.A., decl.) $=\left(134^{\circ}, 3.4\right)$, surrounded by faint, banded substructure roughly parallel to that of the main western stream.

From the reddening map of Schlegel et al. (1998) the maximum values of $E(B-V)$ are $\approx 0.2$ (near the northern tip), with typical values near 0.03 along the remainder of the stream. There are diminutions here and there in the stream that could be attributed to regions of higher reddening, but there are no long features with a north-south orientation that could be held to account for either the appearance of the stream as a whole, or for the different components within it.

Sampling at several representative points, the main stream complex appears to be about $5^{\circ}$ wide on average. This is significantly broader than the globular cluster streams found by Odenkirchen et al. (2003), Grillmair \& Johnson (2006), and Grillmair \& Dionatos (2006a, 2006b). At our estimated distance to the stream (see below), $5^{\circ}$ corresponds to about $800 \mathrm{pc}$, which is much larger than the tidal diameter of a globular cluster. We conclude that the progenitor was considerably more extended than a globular cluster and was most likely a dwarf galaxy.

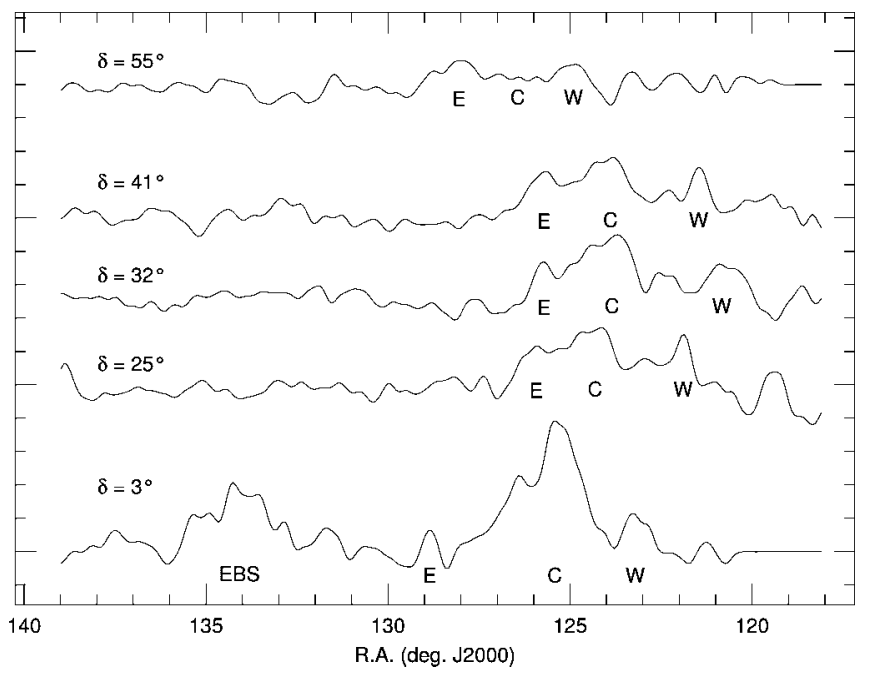

FIG. 2.-Profiles across the stream complex at constant declination, after smoothing the weighted star counts with a Gaussian kernel with $\sigma=0.25$ and summing over 2.4-wide bands in declination. E, C, and W indicate the eastern, central, and western components. EBS denotes the eastern banded structure alluded to in the text. The profiles have been offset vertically for clarity. Note indications of still other streams, both within the central stream and to the east and west of the complex.

The stream is clearly not just a broad swath of stars. Rather, it appears to be made up of a $\sim 2^{\circ}$-wide broad component running roughly along the center of the stream complex, and at least two narrower, $1^{\circ}$-wide streams to the east and west of the broad component. The fine structure in the stream complex is illustrated in Figure 2, where we have made several east-west slices across the complex at various declinations. Comparing Figures 1 and 2, there are indications of additional structure within the broad component and still other, more tenuous parallel streams to the east and west of the three major components.

Integrating the background-subtracted, weighted star counts along the stream over a width of $\approx 5^{\circ}$, we find that the total number of stars in the discernible stream down to $g=22.5$ is $9200 \pm$ 1500. The mean surface density of stars in the southern portion of the stream $\left(-1^{\circ}<\delta<14^{\circ}\right)$ is about 67 stars $\mathrm{deg}^{-2}$, which is roughly twice the $32 \mathrm{stars} \mathrm{deg}^{-2}$ (corrected for $\cos \delta$ ) found in the northern section $\left(39^{\circ}<\delta<52^{\circ}\right)$. The highest local surface densities exceed 200 stars $\mathrm{deg}^{-2}$. If we assume a globular cluster-like luminosity function in the stream, then we can use the color transformation equations of Smith et al. (2002), the deep M4 luminosity function of Richer et al. (2002), and the mass-luminosity relation of Baraffe et al. (1997) to extrapolate to fainter magnitudes. Integrating over $3<M_{V}<17$, we estimate a total number of stars in the visible portion of the stream of $33,000 \pm 5400$, a total luminosity of $5500 \pm 900 L_{\odot}$, and a total estimated mass of $9300 \pm 1500 M_{\odot}$.

\subsection{Color-Magnitude Distribution and Distance to the Stream}

In Figure 3 we show $g, g-i$ color-magnitude distributions for the stream stars, extracted by generating Hess diagrams of stars lying along $2^{\circ}$-wide regions covering the western and eastern halves of the stream complex and subtracting a similar field star distribution sampled over $\approx 500 \mathrm{deg}^{2}$ to the east and west of the stream complex. Despite the somewhat limited statistics, a clear signature of the turnoff and main sequence is evident in the stream population. Moreover, the turnoff regions of the distributions 


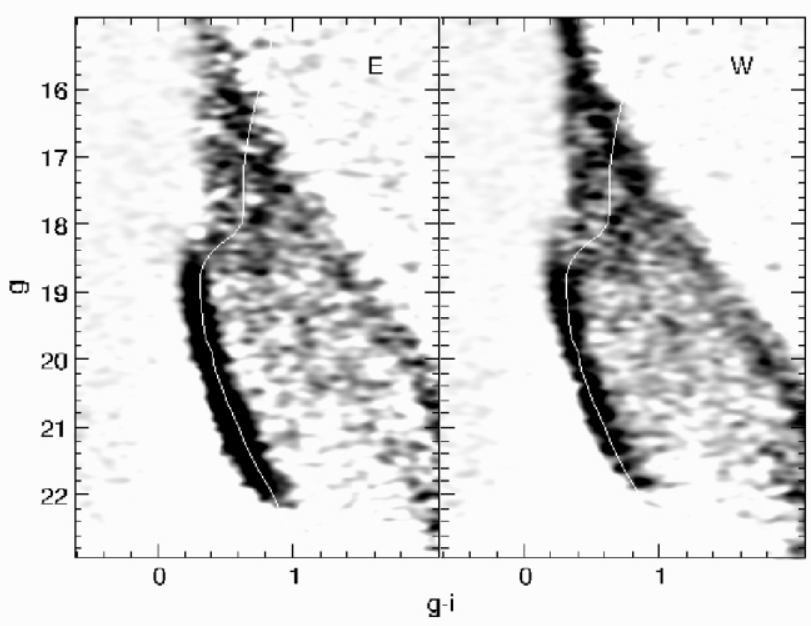

FIG. 3.-Dereddened, background subtracted, color-magnitude distribution of stream stars. The left-hand panel shows the color-magnitude distribution of stars in the western portion of the stream complex, while the right-hand panel shows the distribution of stars in a $2^{\circ}$-wide strip on the eastern side of the complex. The main sequence and the turnoff are clearly distinguishable. The solid line shows the dereddened locus of giant branch and main-sequence stars as measured in DR5 for M13, shifted faintward by $0.3 \mathrm{mag}$.

match the dereddened, shifted main-sequence locus of M13 fairly well. The turnoffs in the eastern and western halves of the complex lie at dereddened $g-i=0.27$ and 0.3 , respectively. A similar diagram in $g-r$ yields dereddened turnoff colors of $g-r=$ 0.23 and 0.25 , respectively, with the difference most likely due to $\sim 0.02$ mag measurement uncertainty. These estimates lie between values of 0.26 and 0.22 measured by Newberg et al. (2002) for the Monoceros Ring and the Sagittarius stream, respectively. They are also much bluer than the Newberg et al. (2002) estimate of dereddened $g-r=0.40$ for thick-disk stars. Solely on the basis of turnoff color, we can therefore rule out association of the stream complex with thick-disk stars. However, within the uncertainties, the turnoff colors are consistent with those of either the Monoceros Ring or the Sagittarius stream.

Varying the magnitude shift applied to M13's main-sequence locus from -1.0 to $+3.0 \mathrm{mag}$, we measured the foregroundsubtracted, mean surface density of stream stars in the regions $-1^{\circ}<\delta<9^{\circ}, 17^{\circ}<\delta<39^{\circ}$, and $39^{\circ}<\delta<63^{\circ}$. To avoid potential problems related to a difference in age between M13 and the stream stars, we used only the portion of the filter with $19.5<g<22.5$, where the bright cutoff is 0.8 mag below M13's main-sequence turnoff. Although this reduces the stream contrast somewhat, it provides sufficient integrated signal-tonoise ratio to enable main-sequence fitting.

Fitting Gaussians to the mean surface densities as a function of magnitude shift (e.g., Grillmair \& Dionatos 2006b), we find that the highest contrasts occur for shifts of $+0.31,+0.29$, and $+0.37 \mathrm{mag}$ for the eastern half of the stream complex over the declination ranges given above, respectively. For the western half, we find that the filter response peaks at shifts of +0.34 , +0.25 , and $+0.38 \mathrm{mag}$, respectively. The magnitude shifts for the two halves are highly consistent with one another, and we conclude that there is no significant distance offset from one side of the complex to the other. Adopting a distance to M13 of $7.7 \mathrm{kpc}$ (Harris 1996) we find an average heliocentric distance of $d=8.9 \pm 0.2 \mathrm{kpc}$. The stream is roughly perpendicular to our line of sight and slightly curved about the Galactic

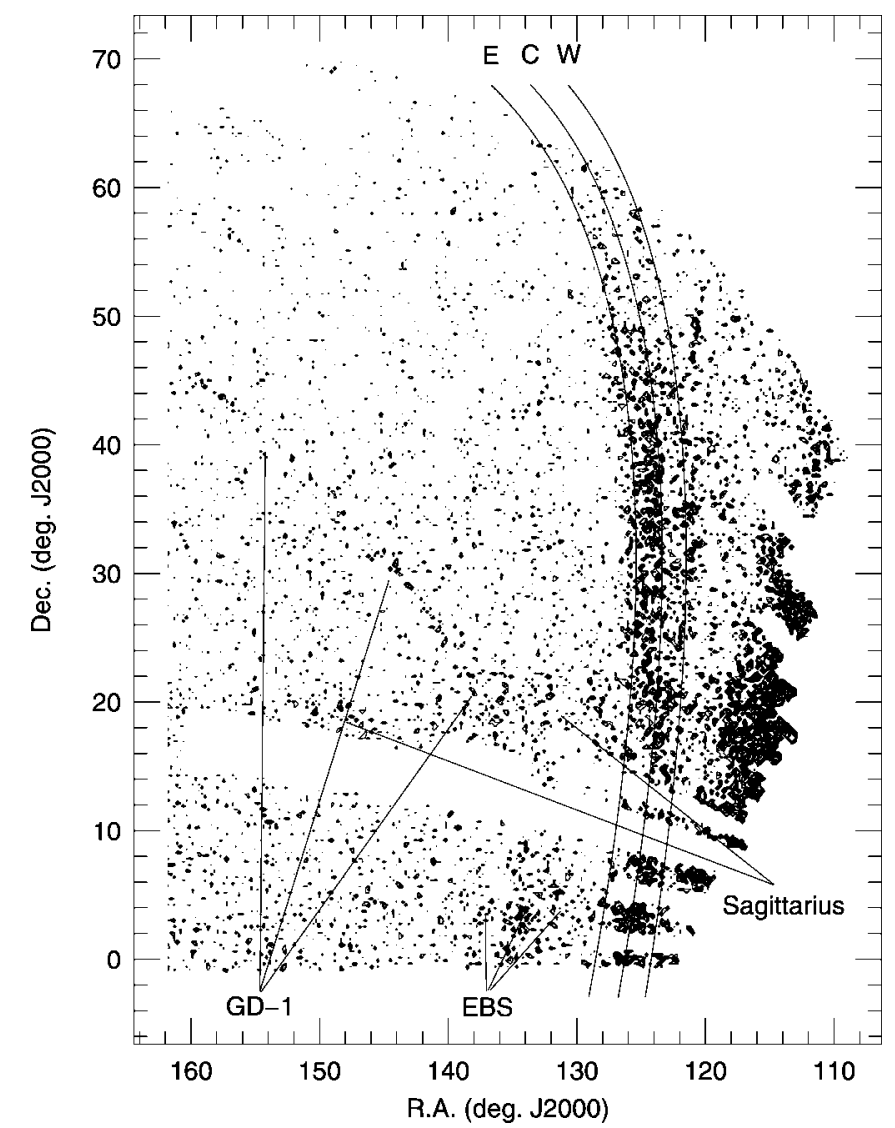

FIG. 4.-Contour plot of Fig. 1, showing projections of the best-fitting orbits to each of the eastern, central, and western stream components. GD-1 is the globular cluster stream found by Grillmair \& Dionatos (2006a) and other designations are as in Fig. 2.

center, as expected. Our distance estimate is in excellent agreement with the $\approx 9 \mathrm{kpc}$ found by Ibata et al. (2003) in their Wide Field Survey (WFS) 0801 field, which is situated on the western edge of the stream complex at $\alpha=120.5, \delta=40.3$.

\subsection{Constraints on the Orbit}

The visible portion of the stream complex spans the Galactic anticenter direction and, projecting a great circle path, is inclined by $35^{\circ}$ to the Galactic plane. Although we are currently limited by a lack of velocity information, for a given model of the Galactic potential the progenitor's orbit is actually fairly well constrained by the observed distance and orientation of the stream. Using the Galactic model of Allen \& Santillan (1991, which includes a disk, bulge, and spherical halo, and which Grillmair \& Johnson [2006] and Grillmair \& Dionatos [2006a, 2006b] found to work reasonably well for NGC 5466, Pal 5, and GD-1), we use a least-squares method to fit both the orientation on the sky and the distance measurements in $\S 3.1$. In addition to a number of normal points lying along the central component of the stream, we chose as a velocity fiducial point a position at the northern end of the stream at (R.A., decl.) $=\left(125^{\circ} .463,51.492\right)$.

If we allow the proper motions to be free-ranging and uninteresting parameters, the model that best fits the data predicts $v_{\text {LSR }}=-18 \pm 10 \mathrm{~km} \mathrm{~s}^{-1}$ at the fiducial point, where the uncertainty corresponds to the $95 \%$ confidence interval. A projection of this orbit is labeled $\mathrm{C}$ in Figure 4 . We note that the 
uncertainty is primarily determined by the large lever arm over which it has been possible to measure relative distances. The $95 \%$ range in $v_{\text {LSR }}$ in turn predicts a range in perigalactic and apogalactic radii of $6.6 \mathrm{kpc}<R_{p}<6.9 \mathrm{kpc}$ and $16.8 \mathrm{kpc}<$ $R_{a}<17.3 \mathrm{kpc}$. Of course, these ranges do not take into account uncertainties in the absolute distance of the stream (which depends on the uncertainty in M13's distance) or of the validity of the Allen \& Santillan (1991) Galactic model.

Given the very similar distances estimated for the eastern and western portions of the stream, it is highly unlikely that the complex could be a superposition of multiple wraps around the Galaxy. The two narrower streams (E and W in Fig. 4) can

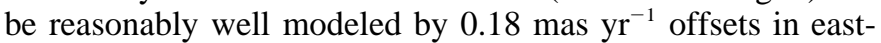
west proper motion. At the distance of the stream complex this amounts to $\approx 8 \mathrm{~km} \mathrm{~s}^{-1}$. The entire stream complex is therefore likely to be the remains of a dwarf galaxy that contained distinct components spanning a range of binding energies. The narrower streams might, for example, be the remnants of the parent galaxy's globular cluster population. Piecing together the original structure and evolution of the stream's progenitor will require detailed $N$-body modeling.

Integrating orbits for parameter sets spanning the range above, we find that, with the exception of the Sagittarius dE, there are no known dwarf galaxies within $5^{\circ}$ of the projected orbit. The Sagittarius dE lies 3:1 from the projection of the best-fit orbit, but the orbital planes of the Sagittarius $\mathrm{dE}$ and the new stream are clearly distinct (Fig. 4). We attribute this apparent proximity to the expected confluence of orbit projections in the direction of the Galactic center and not to any physical association between them.

The orientation of the stream on the sky puts fairly strict limits on the plane of the orbit. The visible portion of the stream passes through Lynx, Cancer, and Hydra. We find no reasonable combination of parameters that would place the southern projection of the stream in either Monoceros or Canis Major. Nor does the visible extent of the stream complex fit either the prograde or retrograde models of the Monoceros stream computed by Penarrubia et al. (2005). Thus, even while the turnoff colors appear to be similar, we conclude on orbital grounds that this stream complex is unlikely to be related to either the Monoceros stream or the Canis Major overdensity.

This work was begun at the 2006 workshop on Deconstructing the Local Group, and the author is grateful to the Aspen Center for Physics for their hospitality. Funding for the creation and distribution of the SDSS Archive has been provided by the Alfred P. Sloan Foundation, the Participating Institutions, the National Aeronautics and Space Administration, the National Science Foundation, the US Department of Energy, the Japanese Monbukagakusho, and the Max Planck Society.

Facilities: Sloan

\section{REFERENCES}

Allen, C., \& Santillan, A. 1991, Rev. Mex. AA, 22, 255

Baraffe, I., Chabrier, G., Allard, F., \& Hauschildt, P. 1997, A\&A, 327, 1054

Belokurov, V., Evans, N. W., Irwin, M. J., Hewett, P. C., \& Wilkinson, M. I. 2006a, ApJ, 637, L29

Belokurov, V., et al. 2006b, ApJ, 642, L137 2006c, ApJ, 647, L111

Grillmair, C. J. 2006, ApJ, 645, L37

Grillmair, C. J., \& Dionatos, O. 2006a, ApJ, 641, L37 2006b, ApJ, 643, L17

Grillmair, C. J., \& Johnson, R. 2006, ApJ, 639, L17

Harris, W. E. 1996, AJ, 112, 1487

Ibata, R. A., Irwin, M. J., Lewis, G. F., Ferguson, A. M. N., \& Tanvir, N. 2003, MNRAS, 340, L21

Majewski, S. R., Skrutskie, M. F., Weinberg, M. D., \& Ostheimer, J. C. 2003, ApJ, 599, 1082
Newberg, H. J., et al. 2002, ApJ, 569, 245

Odenkirchen, M., et al. 2001, ApJ, 548, L165

- 2003, AJ, 126, 2385

Penarrubia, J., et al. 2005, ApJ, 626, 128

Richer, H. B., et al. 2002, ApJ, 574, L151

Rocha-Pinto, H. J., Majewski, S. R., Skrutskie, M. F., Crane, J. D., \& Patterson, R. J. 2004, ApJ, 615, 732

Rockosi, C. M., et al. 2002, AJ, 124, 349

Schlegel, D. J., Finkbeiner, D. P., \& Davis, M. 1998, ApJ, 500, 525

Smith, J. A., et al. 2002, AJ, 123, 2121

Willman, B., et al. 2005, AJ, 129, 2692

Yanny, B., et al. 2003, ApJ, 588, 824

Zucker, D. B., et al. 2006, ApJ, submitted 